\section{GENRE}

en séries

\section{Genre en séries}

Cinéma, télévision, médias

9 | 2019

Cultures juvéniles de l'amour

\title{
Les Thug Love : des romans sentimentaux à l'épreuve de la classe et de la race
}

\section{Mélie Fraysse et Marie-Carmen Garcia}

\section{(2) OpenEdition}

1 Journals

Édition électronique

URL : http://journals.openedition.org/ges/299

DOI : $10.4000 /$ ges.299

ISSN : 2431-6563

Éditeur

Presses universitaires de Bordeaux

Référence électronique

Mélie Fraysse et Marie-Carmen Garcia, «Les Thug Love : des romans sentimentaux à l'épreuve de la classe et de la race », Genre en séries [En ligne], 9 | 2019, mis en ligne le 01 mai 2019, consulté le 18 février 2021. URL : http://journals.openedition.org/ges/299 ; DOI : https://doi.org/10.4000/ges.299

Ce document a été généré automatiquement le 18 février 2021.

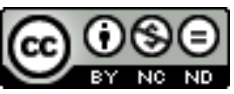

La revue Genre en séries est mise à disposition selon les termes de la Licence Creative Commons Attribution - Pas d'Utilisation Commerciale - Pas de Modification 4.0 International. 


\title{
Les Thug Love : des romans sentimentaux à l'épreuve de la classe et de la race
}

\author{
Mélie Fraysse et Marie-Carmen Garcia
}

Nous remercions Viviane Albenga pour sa lecture attentive et ses suggestions d'améliorations notables de la première version de ce texte.

\begin{abstract}
Les structures de la famille noire ont été conçues comme pathologiques par l'État, tout comme la théorie féministe blanche est en train de les reconstruire. Quelle ironie, que la structure

occidentale de la famille nucléaire, et les idéologies apparentées de "l'amour romantique » développées par le capitalisme, soient vues comme plus « progressives » que les structures de

la famille noire (Hazel, 2008 : 91)
\end{abstract}

1 Les théoriciennes du Black feminism ${ }^{1}$ ont critiqué la centration des analyses féministes sur les pratiques et représentations des groupes sociaux «blancs». Elles ont notamment souligné que ce n'est pas seulement leur subordination sociale en tant que «Noires» qui les différenciait des «Blanches» mais les concepts de «famille», de " patriarcat » de « reproduction » qui ne sont pas adaptés dans les théories féministes à la vie des femmes "noires». La critique intersectionnelle (Crenshaw Wiliams, 2005 [1991]) qui en est issue, évoque - comme nous le voyons dans la citation de Hazel Carby - les représentations normatives de la conjugalité fondées sur le modèle ${ }^{2} \mathrm{de}$ l'amour romantique ${ }^{3}$ en Occident ${ }^{4}$. Élaboré dans les milieux bourgeois du XIX siècle européen (Flandrin, 1982), la représentation dominante de l'amour hétérosexuel est fondée sur la valorisation de l'engagement réciproque, l'éthique du mariage et le « développement de soi » (Shorter, 1977 [1975]). Ce modèle de relations entre les sexes comporte aussi la norme d'égalité (Kaufmann, 1993). Il doit enfin être appréhendé comme une production issue des rapports sociaux à l'œuvre dans le contexte social 
concerné, et non comme un héritage ethnique ou religieux. Plus précisément, "l'imbrication du sexisme et du racisme constitue le contexte social à partir duquel ces normes [amoureuses et sexuelles] sont élaborées » (Hamel, 2006 : 56).

Nous proposons dans ce texte une analyse du modèle amoureux dominant occidental dans des productions culturelles élaborées par et pour des jeunes filles racisées en France. Les groupes "racisés» sont des groupes altérisés en fonction d'une caractéristique «somatique non changeable» (Guillaumin, 1972). Toute catégorie sociale pensée comme "biologiquement" différente peut ainsi faire l'objet d'une racialisation ("femmes», "jeunes», "vieux »...). Il s'agit d'un processus de construction de l'altérité : processus (politique, pratique, discursif) par lequel des individus se considèrent semblables (ou par lequel des groupes sont supposés homogènes) et instaurent ou confirment des distances sociales pensées comme irréductibles avec d'autres individus ou groupes (Hammouche, 2013). L'utilisation du vocable «altérité » a pour objectif ici d'insister sur le caractère construit du groupe social désigné habituellement par l'expression «issu de l'immigration $»^{5}$. Nous nous focalisons ainsi sur un sous-espace à l'intérieur du sous-champ ${ }^{6}$ de la littérature populaire sentimentale qui n'a pas encore été exploré : des chroniques qui apparaissent depuis quelques années en France sur Internet appelées Thug Love.

3 Le terme "Thug » est un acronyme inventé par Tupac Shakur, un célèbre rappeur américain mort assassiné. L'expression désigne une certaine catégorie de voyous. Il signifie "The Hate U (you) Give» («La haine que tu transmets»). On parle de "Thug Life » pour désigner le mode et code de vie de certains voyous dans les ghettos américains et dans le langage des cités ${ }^{7}$. Il est difficile de faire une histoire de cette thématique mais il semble que les termes "Thug love » apparaissent en France tout d'abord dans la musique et plus particulièrement dans les paroles de chansons de rap ou de R'n'B dans la première décennie des années 2000. C'est la chanteuse Kaylia qui dans son album "Caractère » publie une chanson baptisée " Thug Love » et va diffuser ce terme et cette thématique de façon massive. Elle revendique dans ce morceau, de faire souffrir son amoureux comme il l'a faite souffrir et elle menace ce dernier de faire la Thug en cas de rupture :

« Tu veux me quitter, y'en a qui rêvent d'avoir ta place toi-même tu sais »

«Juste un coup de fil et j'te remplace » [...]

"C'est le thug love y'a plus de love story "

«Tous les coups sont permis"

4 Les Thug love en tant que récits apparaissent de façon importante dans les années 2010 même si quelques chroniques précoces sont publiées sur skyblog dès 2008. Écrites $a$ priori par des jeunes filles "des cités » de par les pseudonymes utilisés ainsi que les prénoms des protagonistes, ces chroniques mettent en scène des amours tumultueuses de jeunes filles "de bonne famille" avec de "mauvais garçons" (se livrant à des activités illicites : trafic de drogue, recel, braquage etc...). Qu'ils s'apparentent à du témoignage ou de l'autofiction, ces récits sont publiés sous forme de chroniques et paraissent quotidiennement principalement sur deux (la troisième étant Facebook) plateformes de publication numériques en ligne: Wattpad et Skyblog. Certaines chroniques sont achevées, d'autres évoluent encore et ce, depuis plusieurs années (2 ans). Un décompte rapide donne une estimation de 300 histoires publiées pour Skyblog et 400 pour Wattpad. Le nombre d'abonné-es varie entre 15 et 500, la moyenne se situant vers 50. Ces chiffres ne concernent que la période couverte par l'étude (depuis 2016), mais entre 2010 et 2014, les chroniques les plus populaires comme «Cendrillon 
amoureuse d'un prince du ghetto " ou "Chronique d'une Cendrillon promise à un prince de Tess » (publication Wattpad et Facebook) ont pu avoir jusqu'à 50000 fans. Plus que le nombre de chroniques publiées ce sont ces chiffres sur les fans qui permettent de qualifier ces quatre années comme l'âge d'or des Thug love sur internet. Deux d'entre elles ont fait l'objet d'une publication papier: «Dans la peau d'un Thug " de Nargesse Bibimoune, IS Editions, 2013 et «Entre amour et raison » de Laila L. Edilivre, Tome 1, 2013- Tome 2, 2014.

5 Les Thug love sont ainsi une forme culturelle. Nous considérons ici que «le modèle de l'autonomie symbolique, de quelque manière qu'on le compose avec le modèle de la domination symbolique n'est jamais disqualifié d'avance dans le travail de description du sociologue, sauf à abdiquer par principe (éthique ou politique) toute analyse culturelle dans le cas des cultures populaires $"{ }^{9}$. Comme toute forme culturelle, les Thug Love connaitraient donc des variations, des modalités d'expression, de production et d'appropriation à la fois "dominées» et "autonomes » par rapport au roman sentimental édité.

Plus précisément, il s'agit ici de suivre la ligne théorique ouverte par Roger Chartier (1988) concernant la notion d'" appropriation" des textes. L'historien propose de dépasser l'antagonisme entre les approches en termes de " consommation culturelle » et celles en termes de "réception culturelle ». La première provient principalement des travaux de Pierre Bourdieu (1979) et se fonde sur la thèse d'un accès socialement différencié aux productions culturelles. La seconde, initiée par Richard Hoggart (1970), propose de traiter les pratiques de lecture et d'écriture des classes populaires non conformes aux codes culturels légitimes comme des formes de résistance subversives à la culture dominante. L'approche de Roger Chartier conduit à saisir les lignes de partage entre lectures différentes d'un même texte en considérant qu'il existe "une pluralité raisonnée, et non pas une infinité, de lectures possibles d'un même texte " (Albenga, 2017 : 19). Les variations sociales d'appropriation des textes ne peuvent, en outre, être réduite aux seules appartenances de classe: «L'histoire sociale a trop longtemps accepté une définition réductrice du social, confondu avec la seule hiérarchie des fortunes et des conditions, oubliant que d'autres différences, fondées sur les appartenances sexuelles, territoriales ou religieuses, étaient elles aussi pleinement sociales et susceptibles de rendre compte, autant ou mieux que l'opposition dominants/ dominés, de la pluralité des pratiques culturelles» (Chartier, 1987: 9-10). Cette approche de l'appropriation culturelle en contexte populaire (Harchi, 2018) permet de penser que nous avons affaire, avec les Thug love, à un réinvestissement socialement orienté de la forme culturelle que représente le roman sentimental. Les Thug love constitueraient, selon cette hypothèse, une modalité spécifique d'appropriation de ce dernier.

7 Nous suivons alors l'idée qu'en tant qu'objet culturel doublement dominé (dominé à l'intérieur d'un sous-champ littéraire lui-même dominé) mais aussi relativement autonome, les Thug love mettent en récit des normes et des valeurs autonomes et hétéronomes par rapport au roman sentimental populaire (Bettinotti, 1986; Coquillat, 1988 ; Constans, 1999 ; Helgorsky, 1985 ; Péquignot 1991) dont la collection Harlequin constitue une des principales références ${ }^{10}$. Celui-ci se définit par «une intrigue structurée autour de la relation entre un homme et une femme, dont l'enjeu est le jeu des passions, des sentiments et la quête, sans cesse retardée par des obstacles, de l'amour et du bonheur, réalisés par la fusion de deux entités en une » (Damian-Gaillard, 
2014 : 320). Le roman Harlequin est partie prenante de la "culture de masse " (La Mothe, 1989) et contribue aux socialisations sentimentales féminines (Houel, 1997). En somme, il est populaire et féminin.

8 Les chroniques étudiées mettent, elles, en scène, des héroïnes et des héros absent.e.s de la littérature populaire (Hoggart, 1970): des jeunes filles et des jeunes garçons de familles originaires d'Afrique du Nord ou de Turquie et vivant dans des " cités ${ }^{11}$. Elles mettent en scène des histoires d'amour « impossibles » notamment parce que le héros est un "mauvais garçon» et l'héroöne une fille de "bonne famille». Nous nous attacherons prioritairement aux images de la ou des masculinité(s) et féminité(s) ${ }^{12}$ musulmanes en milieux populaires ${ }^{13}$ proposées dans ces romances. La première partie du texte sera consacrée au modèle amoureux et la seconde au modèle de genre de ces romances en ligne.

\section{Encart méthodologique}

Les textes étudiés dérogent constamment aux usages légitimes du français, de l'écriture et de la narration. L'orthographe, la syntaxe et la structure des textes montrent que l'on a affaire à un langage en rupture avec la culture scripturale scolaire. Quatre chroniques ont été choisies, sélectionnées dans les deux plateformes de publications numériques : Skyblog et Wattpad. Les critères de choix portaient principalement sur le nombre de pages (entre 100 et 200) mais aussi sur des publications terminées.

Cette enquête s'est déroulée en deux temps. Nous avons tout d'abord effectué une étude de corpus en "récupérant» les données sur Wattpad et Skyblog par webscraping afin de poursuivre par une analyse lexicométrique puis thématique à l'aide du logiciel Iramuteq ${ }^{14}$, permettant le traitement d'une masse importante de données. La méthode utilisée dans Iramuteq était celle de Reinert (Marpsat, 2010). Les unités de contexte initiales, (UCI), représentaient les différents blogs et les unités de contexte élémentaires (UCE) des segments de texte de longueur de 40 mots, les formes réduites actives correspondant aux adjectifs, noms communs, adverbes et verbes (les adjectifs démonstratifs, indéfinis, les articles, les auxiliaires, les chiffres, les onomatopées, les pronoms ne sont pas utilisés pour mener l'analyse). Le but de la classification était de réunir les UCE en un nombre de classes réduit, les UCEs étant rapprochées suivant leur proximité lexicale (Marpsat, 2010). Ici, six classes de mondes lexicaux ont été identifiées :

- Classe $1:$ Les activités extérieures

- Classe 2 : Les descriptions physiques

- Classe 3 : Les relations amicales

- Classe 4 : Les relations avec la famille

- Classe 5 : Les relations sexuelles

- Classe $6:$ Les activités intérieures

11 À la suite à cette première analyse, il est apparu que les classes 1 (activités intérieures), 2 (descriptions physiques) et 5 (relations sexuelles) étaient les plus représentées dans l'intégralité du corpus. Elles constituaient les «thématiques » étudiées dans ce travail de par ce caractère "saillant ». À la suite de cette analyse quantitative, une analyse qualitative a pu être effectuée notamment pour étudier la relative distance/proximité 
avec les romans de genre sentimental dont Harlequin constitue l'exemple le plus représentatif.

\section{L'expression d'une intériorité au féminin}

12 Nos analyses montrent prioritairement une forte proximité narrative des Thug love avec les romans Harlequin étudiés par Béatrice Damian-Gaillard (2011). D'abord, comme dans ces romans sentimentaux "grand public ", on a affaire à une littérature réflexive. Elle est centrée sur l'intériorité des actants selon un " procédé de focalisation interne » (Olivier, 2009) qui donne le point de vue des héros et héroïnes. Comme dans les romans sentimentaux populaires, dans les Thug love, le point de vue adopté est celui de l'héroïne. C'est l'héroïne qui raconte l'histoire et qui se questionne sur ses amours. Ce type de récit semble constituer une appropriation populaire des écritures intimes inaugurées par les femmes bourgeoises du XIX ${ }^{\text {ème }}$ (Lacoue-Labarthe et Mouysset, 2014). Ce texte écrit au féminin se présente ici sous un double aspect. D'une part, par le procédé d'écriture même, il est sensé révéler les pensées, les émotions, les sentiments des auteur.es ${ }^{15}$ (héroïnes du récit). D'autre part, il produit des normes morales et des pratiques pouvant contribuer à l'élaboration du « for intérieur féminin » des lectrices (Charpentier, 1995). D'ailleurs, l'argot fréquemment utilisé, le « langage de la rue » ou l'usage de mots et expressions arabes favorisent le " contrat de lecture » et conduisent a priori les lectrices à "retrouver du semblable» (Bigey et Olivier, 2010) dans ces fictions comme en témoignent les commentaires postés sur les blogs ${ }^{16}$.

Layal : J'avais honte d'avoir déçu ma famille de m'être fait jeté de cette manière j'en voulais à Aymen mais malgré tout je l'aimais encore l'amour que j'éprouvais à son égard était indescriptible. Je pense que vous avez toutes déjà vécu sa un amour si fort que quoiqu il fasse malgré ses erreurs tu lui porte un amour de plus en plus fort chaque jours.

Chronique d'Aymen et Layal : les opposés s'attirent (Parisienne, Wattpad, 2015).

Ensuite, les chroniques amoureuses sur internet disposent d'un référent sociosémiotique et rhétorique renvoyant à un univers perçu comme féminin. A l'instar des invariants des romans d'amour de la collection Harlequin (Bettinotti, Noizet, 1989), l'histoire est toujours racontée du point de vue de l'héroïne qui interroge ses amours et ses désirs, sujets principaux du récit. Le scénario est également stable de type «boy meets girl».

\section{Des catégories ethnico-religieuses en toile de fond}

14 Malgré le caractère sériel et très stéréotypé du scénario, les romans Harlequin comportent quelques variations dans les intrigues amoureuses. En effet ces dernières se déroulent parfois ailleurs que dans les pays occidentaux où elles sont majoritairement installées. Certaines histoires se passent ainsi à d'autres époques (collection «les historiques »), dans d'autres pays (la Scandinavie, un royaume d'Arabie), avec des héros masculins a priori distanciés de l'homme blanc et urbain. C'est le cas du " héros arabe " et de la série "Orientale». II s'agit en fait de "l'Arabe $~^{17}$ en tant que catégorie construite de l'altérité ethnique et raciale (Rea, Tripier, 2008), autrement dit en tant que conceptualisation de la réalité sociale élaborée à l'intérieur de luttes de classement visant à la reconnaissance d'un groupe ethnico-culturel considéré comme fondamentalement "étranger " aux valeurs occidentales (dominantes). Cette catégorie 
de l'altérité («l'homme arabe ») s'inscrit à l'intérieur du savoir moyen supposé (par l'éditeur) de la lectrice cible du roman Harlequin.

La représentation stéréotypée de "L'Arabe ", comme figure narrative, montre celui-ci comme brutal, sauvage, jaloux et polygame. Cette représentation de "l'homme arabe " ne concerne pas seulement - peut-être pas prioritairement - une stigmatisation des hommes orientaux en Occident (Macé, 2007), mais une image des femmes en Orient (dominées, asservies...) qui légitime le contrôle des femmes dans les pays occidentaux : «En Occident, les arguments du développement économique confortent l'idée que, avec l'extension des systèmes d'éducation occidentaux, la modernisation des forces de travail et le renforcement de l'individualisme, les femmes seraient libérées de la domination d'hommes gouvernés par les valeurs patriarcales traditionnelles " (Nader, 2006: 18). En effet, souligner les pratiques différenciant les femmes selon leurs « appartenances » contribue à amalgamer les violences envers les femmes et le groupe minoritaire d'une part et l'égalité entre les sexes et le groupe majoritaire d'autre part (Hamel, 2006). Les représentations sociales à l'intérieur du groupe dominant de la condition des femmes participent ainsi du renforcement de la différenciation et de la hiérarchisation symbolique, politique et idéologique de type " nous/eux ».

Cela dit, les auteur.e.s Harlequin doivent respecter le motif final, qui est le mariage, de type strictement monogamique, impliquant la fidélité mutuelle. L'héroïne Harlequin ne pourra jamais être amoureuse d'un Arabe qui ne serait pas d'une classe sociale supérieure, hautement diplômé et présentant les attributs moraux et physiques du Prince Charmant (le héros est par exemple un émir, il parle français ou anglais parfaitement, son père ou sa mère sont d'ailleurs de l'une de ces nationalités, il est diplômé d'Oxford etc.). Le roman ne peut pas non plus déroger au contrat de lecture qui veut que les deux protagonistes convolent en justes noces et qu'un avenir idyllique se dessine devant eux.

Dans les Thug love, les auteur.e.s des chroniques amoureuses illustrent leurs récits avec des prénoms et du vocabulaire d'origine magrébine ou turque. Elles usent ainsi de catégorisations construites de groupes ethniques minorisés ou du statut d'immigré qui renvoient à la figure de l'Autre, ici « le Turc » ou «l'Arabe».

Présentation de Sherazade: Je me présente Sherazade, d'origine turk \& algerienne \& j'en suis fiere, j'ai 17 ans, je suis la plus petite de ma familles, j'ai 1 grand frére qui s'apelle Mohamed il a 19 ans

Chronique de Sherazade, (Sherazade, skyblog, 2013).

Présentation de Layal: salam moi c'est layal19 ans je suis plutôt petite de taille moyenne avec des formes sans vouloir me vanter mdr j'ai les yeux marron très clairs c'est vraiment choquant on me fais toujours la remarque sur mes yeux al hamdoullilah je vis à montréal toute seule je travaille dans un hôtel en tant que réceptionniste j'ai fais des études hôtelières après le brevet c'est un hôtel très connu donc ça paye assez bien même très bien. Mes parents sont a bamako ah oui je suis libanaise et malienne mélange bizarre hein!

Chronique d'Aymen et Layal : les opposés s'attirent. (BangOCarre, Wattpad, 2014).

\begin{tabular}{|l|l|l|l|l|}
\hline Titre de la chronique & $\begin{array}{l}\text { Noms des } \\
\text { protagonistes }\end{array}$ & Origines & $\begin{array}{l}\text { Support de } \\
\text { publication }\end{array}$ & Pseudo \\
\hline
\end{tabular}




\begin{tabular}{|l|l|l|l|l|}
\hline $\begin{array}{l}\text { Chronique d'Aymen et } \\
\text { Layal: les opposés } \\
\text { s'attirent. }\end{array}$ & Aymen et Layal & $\begin{array}{l}\text { Aymen: } \\
\text { Tunisie et } \\
\text { Maroc } \\
\text { Layal : Mali et } \\
\text { Liban }\end{array}$ & Wattpad & BangOCarre \\
\hline Kidnappée par un Thug & Sanaa et Youness & Algérie & Wattpad & Parisienne \\
\hline Chronique de Shérazade & $\begin{array}{l}\text { Shérazade et } \\
\text { Zaid }\end{array}$ & $\begin{array}{l}\text { Turquie et } \\
\text { Algérie }\end{array}$ & Skyblog & Shérazade \\
\hline Ghetto love chronique & Mélina et Amir & Turquie & Skyblog & Ghettolovechronique \\
\hline
\end{tabular}

Cela dit, alors que "l'homme arabe » de Harlequin présente des attributs socialement valorisés dans la représentation romantique de l'amour, les Arabes et Turcs des Thug Love sont de basse condition, n'ont pas suivi d'études et ne présentent, d'une manière générale, aucune des caractéristiques de la romance sentimentale, ni du conte de fées. Ils sont violents, trafiquants de drogues, délinquants ou truands. Ils adhèrent aux valeurs de la "culture de rue » et des gangs. Les figures féminines des chroniques internet tiennent, quant à elles, des positions sociales plus élevées que les hommes. Elles sont issues de familles aisées et ont suivi des études.

Mais elles se différencient fondamentalement des héroïnes des romans sentimentaux par leur personnalité séductrice, manipulatrice et tentatrice. L'héroïne manipule, en effet, le héros grâce à son corps très érotisé, tout en restant vierge. Ce dernier point est crucial dans la structuration du récit amoureux. La préservation de la vertu de l'héroïne, selon les préceptes de l'islam est régulièrement rappelée pour nuancer les propos érotiques qui parsèment les descriptions des rencontres entre les héros. Les interdits religieux sous les termes « hlel» (pure, conforme aux préceptes de l'islam, ce qui est permis) ou "hram» (l'impur, ce qui est interdit), sont ainsi fréquemment mobilisés dans le texte pour les actions mettant en jeu le corps ou «les choses de l'amour ». Il s'agit de "sauver l'honneur " tout en accédant à une certaine liberté sexuelle. Les auteur.e.s mettent ainsi en récit des tensions sociales dont les Françaises ayant des parents issus de l'émigration turque ou maghrébine sont porteuses : «la vie moderne ayant pour conséquence une liberté sexuelle et la vie traditionnelle ayant pour préalable le maintien de la virginité comme moyen d'accès à la vie sociale " (Ferhati, 2007 : 175). Les héroïnes sont alors tentatrices et séductrices tout en insistant sur l'importance de la préservation de leur virginité, comme dans cette scène où apparaissent Aymen et Layal :

Il $m$ embrasse mais ap un bisou normal il ma manger wesh $m d r d u$ coup je vois dans ses yeux qu il veut $s$ arrêter mais je sais pas on dirait il est excité mais je vais ap le laisser me violer mdr il me regarde chelou se lève et part

il est chelou lui wesh ces pas ma faute je veux rester gevier jusqu_à mon mariage wesh du coup je me lève je vais le rejoindré il est sur le canapé du salon.

Chronique d'Aymen et Layal : les opposés s'attirent. (BangoCarre, Wattpad, 2014)

Nous retrouvons également l'attitude "vertueuse » des héroïnes dans les chroniques, sous la forme de rendez-vous "cachés » dans la cité, de messages par sms ou de contacts par personnes interposées : ami.e.s. Pour Zaid et Sharazade les rendez-vous se font par exemple sous les abris bus, ils s'embrassent mais ne vont « jamais plus loin ». 
Rendez-vous de Zaid et Sherazade :

Il est venue preés de moi avec son beau sourire laah wallah tu crake devant son sourire, et il ma fait un bisou sur le front ! ouahhhhhh la je peut vous dire que je suis montéé au 7eme ciele, je sais y'en qui von petetre ce dire c qu'un bisou,mes je vous rassure que de la part de Zaid ,ZAID le thug ! c'est deja beaucoup !

Chronique de Sherazade, (Sherazade, skyblog, 2013)

La tension érotique mise en scène au travers de descriptions de corps nus ou dénudés et par des images suggestives n'est donc jamais envisagée du point de vue de l'impur; le « hram » étant invoqué uniquement quand la virginité de la jeune fille est questionnée. En d'autres termes, les protagonistes "dorment ensemble", se "frottent l'un à l'autre » sans éprouver du remord, mais ne font pas l'amour, cette étape (et celle-ci uniquement) étant qualifiée d'impure, surtout pour l'héroïne.

Déclaration d'Amir à Mélina : Nouveau message de " Amir " : Tu sais Mélina j'ai eu des relations avec des ta de meufs que tu peux meme pas imaginer sa dois faire x100 plus que toi, j'ai couché avec, j'ai fai des choses que jpeu meme pas texpliquer. J'ai meme fait des concours celui qui foure le + de meufs en un seul jour jen avais 4 jpouvais faire ce que je voulais avec la meuf que je voulais. Mais un jour jtai tomber sur une meuf jla kiffé dur et elle ma trompé ( oui il sourit tous le temps et pour rien , c'est pour cacher son cote sensible il m'semble. ). Jme suis dis cs pas pr rien c un retour des flammes. Jme suis dit pk autan vouloir coucher avec plein dmeuf alors ke jpourrai essayer dme poser tkl avc une meuf serieuse. Dpui ce jour sa fai 2 ans j'ai pas trouvé de meuf serieuse et mn max avc une meuf ct 5jours meme pas et mtn jme di jai enfin trouver une meuf que jaime vrm pk la perdre quand tme dis que jte prend pour un delire jme demande pk je t'aime ...

Ghetto love chronique (Ghetto love chronique, skyblog, 2012).

Si le héros a des relations sexuelles avec des filles qu'il qualifie de "keh ou kehba » c'est-à-dire des prostituées, uniquement "pour se soulager", l'héroïne elle, reste vierge. Cette catégorisation symbolique des personnages féminins : celles incarnant des figures féminines connotées négativement, à travers la prostitution (réelle, métaphorique, virtuelle...) et celles considérées comme positives à travers la «féminité mascarade » (paraître féminine...) ou le maternalisme (dévouement à la maternité...) (Lemoine-Luccioni, 1976) n'est pas propre à ces productions culturelles. Elle constitue un socle puissant de l'ordre de genre établi (Pheterson, 2001[1996]) que nous trouvons ici mis en scène à travers des fictions. Sa présence saillante dans les chroniques conduit à nuancer les modalités de transgression de l'organisation dominante du genre (Connell, 2014) que la dissymétrie entre les positions sociales des héros (la femme est diplômée et de classe sociale élevée, l'homme est de «basse extraction») pourrait laisser entrevoir.

\section{Le genre des thugs love}

\section{Ambivalence de la transgression de l'ordre de genre}

La proximité entre thug love et romance de type Harlequin se situe ainsi principalement dans la structure du récit. Mais les deux types de récits se différencient également dans les portraits du héros et de l'héroïne, ou autrement dit dans ce que Julia Bettinotti et Pascale Noizet (1989) définissent comme les motifs variables. En effet, les héros Harlequin (Coquillat, 1988) et Thug sont tous deux plus vieux que la jeune fille, beaux, musclés, amers (porte une faille en eux), machos, misogynes et brutaux et en position de pouvoir matériel et décisionnel. Mais le héros Thug est dominé par 
l'héroïne du point de vue du capital culturel et scolaire. En d'autres termes, si le héros Harlequin est un prince souvent diplômé d'une grande université et parlant plusieurs langues, le héros Thug est un trafiquant de drogue (parfois à l'échelle internationale), ayant quitté l'école précocement. Le portrait de l'héroïne diffère entre Harlequin et Thug love de façon saillante. L'héroïne Harlequin (Coquillat, 1988) est une jeune fille très belle, souvent issue de la classe favorisée. Mais elle est peu sûre d'elle, ignorante de ses droits et des choses de l'amour, à l'image des oies blanches du XIX ${ }^{e}$ siècle (Knibiehler et al., 1983). À l'inverse, l'héroïne Thug est en position de domination sociale et intellectuelle par rapport au héros (langage et bonne manière de la jeune fille vs ceux du jeune homme). Elle est également ambitieuse, manipulatrice, capricieuse et elle est décrite de façon récurrente comme particulièrement « sexy ».

\section{Le genre racialisé}

L'identification «arabe » revendiquée dans les récits en ligne semble renforcée par la défense du principe de virginité qui, pourrait, comme l'explique Christelle Hamel, relever d'un processus de valorisation d'une identité stigmatisée. Le pendant négatif des filles «vertueuses" est constitué par des filles «faciles» qui, dans les récits, ne sont ni héroïnes, ni valorisées. Ces figures « négatives » tiennent un rôle de faire-valoir des héroïnes dans la mesure où ces dernières sont d'autant plus valorisées qu'elles sont belles, intelligentes, séduisantes mais qu'elles « ne cèdent pas à la tentation » de faire l'amour avec leur héros. Ici encore, si l'on suit les analyses de Christelle Hamel, on peut suggérer que l'on a affaire à une "accusation de traîtrise envers les femmes maghrébines qui adoptent le discours des 'Français.e.s' «(Hamel, 2006 : 48). Dans cet extrait Youness le Thug de la chronique explique à Sanaa, jalouse, que Soukaïna, une fille qui « couche » est considérée comme une pute et a donc un statut inférieur à elle. Sanaa peut dormir avec Youness même s'ils ne font pas l'amour, alors que Soukaïna avec qui il a des relations sexuelles, n'a pas le droit de dormir avec lui.

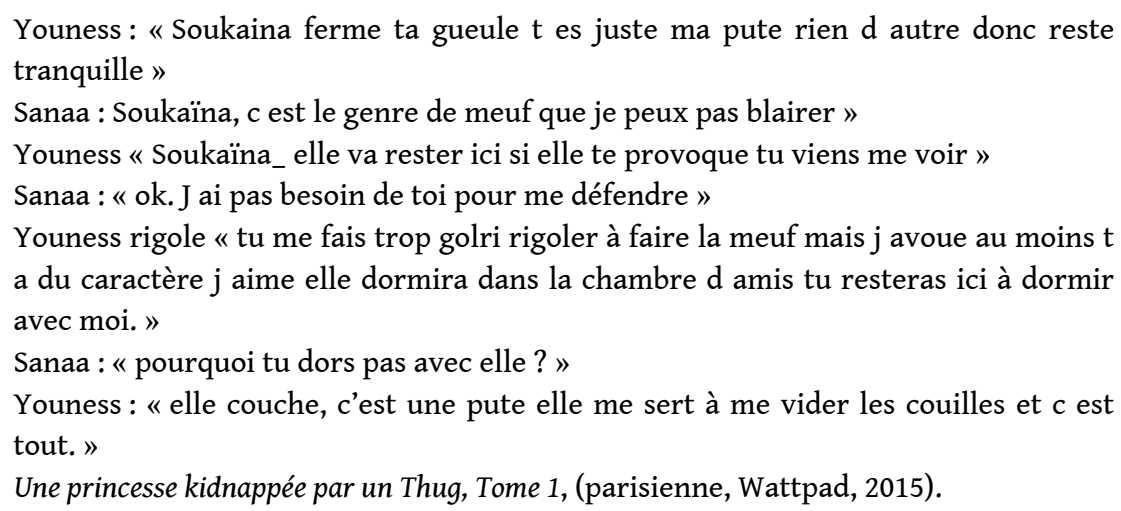

L'effort réalisé par les héroïnes pour «faire entrer dans le droit chemin» leur amoureux délinquant constitue un élément supplémentaire de valorisation des héroïnes. La présence féminine est alors présentée comme positive dans la mesure où elle permet de «canaliser » la violence des garçons. Ce rôle de "normalisation » des conduites masculines donné dans le texte aux femmes fait écho aux attributs dominants de la féminité dans l'ordre social établi. En effet, socialement, l'idée que les hommes répondent à des "pulsions" dont les femmes doivent tenir compte dans chacun de leurs gestes, lorsqu'elles s'apprêtent, lorsqu'elles bougent ou restent immobiles est une des clefs de voûte du système de genre (Coutras, 2003; Clair, 2007 ; 
Jamain-Samson et Liotard, 2011). Dans les Thug love, la figure de la « femme canalisant l'homme" se présente sous les traits de l'héroïne qui met comme condition à la poursuite de la relation amoureuse l'abandon de la vie de Thug. Dans l'extrait suivant de la chronique Kidnappée par un Thug, Youness explique dans un long monologue pourquoi il s'est «rangé » (il a arrêté le hram) de façon à plaire à Sanaa l'héroöne de l'histoire.

J'ai toujours pas demander sa main à ses parents vous savez pourquoi parce- que ça fait 1 ans que y a que Sanaa dans ma tête cette meuf je vous jure c'est à cause d'elle que j'ai arrêté le hram. Cette nuit ou elle m'a dit « toi et moi c'est impossible » j'ai compris que jamais je trouverais une meuf avec cette situation j'ai monté mon entreprise aujourd'hui je suis directeur. En 1 ans je me suis reconstruit chaque jour en pensant à elle bat les couilles si ça fait pd ce que je dis la seule chose que je sais c'est que je l'aime.

Une princesse kidnappée par un Thug, Tome 2, (parisienne, Wattpad, 2015).

Le modèle occidental de la sexualité (la "liberté sexuelle », les relations sexuelles hors mariage...) différencie ainsi les Thug love des romances Harlequin. En effet, d'abord, le principe de virginité n'apparaît pas explicitement dans ces dernières, ensuite, depuis quelques années, Harlequin a élargi ses collections en intégrant une série de livres érotiques : "Des romans d'amour où passion, audace et sensualité se rencontrent. La collection Audace vous plonge dans un univers intense et sexy. Osez la romance érotique! ». Les scènes décrivent par exemple des pénétrations ou des pratiques BDSM. Même si cette collection ne constitue pas le fleuron d'Harlequin, les romans populaires ont de toute évidence investi le marché des romans érotiques.

Dans les Thug love, les héroïnes stigmatisent pour elles et non pour le héros les relations hors mariages. Celles-ci sont en revanche plutôt valorisées pour les garçons en leur conférant un statut de dominant, notamment par rapport aux autres personnages masculins de la chronique. En d'autres termes «l'innocence perdue, qui dévalue les filles, est propre à rehausser le statut des garçons. Le sexe et la violence déshonorent les femmes mais font l'honneur des hommes. [...] La honte de la femme est l'honneur de l'homme. » (Pheterson, 2001 : 110). Cette fidélité affirmée aux valeurs de la " communauté » fait échos aux stratégies de négociation interculturelle de jeunes femmes qui obtiennent, au moins tacitement, la possibilité de prendre des distances avec le code de comportement ancestral à condition d'affirmer leur fidélité aux valeurs considérées comme emblématiques par le groupe (Gaymard, 2003). Ainsi, la question sexuelle ne peut, dans ce corpus, être abordée que sous couvert d'une vertueuse protestation d'innocence : au fond, les valeurs morales ne sont pas vraiment mises en cause. Les chroniques montrent ainsi une acceptation tacite de la domination masculine dans laquelle la femme est un « être perçu » soumis à une double contrainte de retenue et de séduction (Bourdieu, 1990).

\section{Conclusion}

Mettre en parallèle les romans sentimentaux et les Thug Love, permet au final d'observer comment, dans un espace culturellement dominé (les chroniques internet), à l'intérieur même d'un sous-champ dominé du champ littéraire (les romans sentimentaux), des normes de genre racialisées sont produites et reconfigurées. On observe des proximités entre les romans sentimentaux et le récit sur internet notamment en ce qui concerne le type de récit: centration sur le «for intérieur » 
féminin et amour «impossible». Le point convergent de ces romances est la légitimation de la domination masculine. Celle-ci prend des formes différentes selon le support et on observe que l'asymétrie, en faveur des personnages féminins, des positions socio-culturelles dans le couple fictionnel constitue une variation des modalités de mise en scène de la relation amoureuse qui, in fine, est à dominance masculine. Les souffrances féminines mises en récits en attestent. En effet, les premiers temps d'une relation amoureuse hétérosexuelle sont marqués par une ambivalence de la reconnaissance réciproque (construire le "nous amoureux", considérer que l'on « est en couple ») entre les partenaires : les hommes tendent à défendre leur autonomie et à ne pas "se sentir engagés » alors que les femmes œuvrent patiemment et dans la douleur à la construction du couple qu'elles désirent, quelle que soit leur position sociale (Castrillo Bustamente, 2018). Harlequin et Thug love mettent en scène ces souffrances qui valorisent l'abnégation féminine et légitiment la violence masculine. En somme, dans les Thug love, la relative dominance sociale féminine (les femmes détiennent des positions sociales supérieures aux hommes) est mise au service des hommes qu'elles aiment. En revanche, les récits Thug love se distinguent des romances Harlequin en ce qui concerne les normes et les valeurs amoureuses. Les récits sur internet proposent en effet des histoires qui «ne finissent pas toujours bien», le mariage ne constitue pas la finalité de la romance. Les amours « impossibles » mis en récit ne semblent pas destinés à devenir " possibles ", autrement dit à donner naissance à un couple installé. On constate également qu'une des thématiques centrales est la préservation de la virginité de l'héroïne. Cette dernière est ainsi en congruence avec un modèle socialement valorisé des jeunes filles racialisées : une féminité érotisée associée au respect du principe de virginité.

Plus largement, l'éloignement de la structure des Thug love et des romans Harlequin tient à la mise en récit d'une vision de l'amour en rupture avec les normes et valeurs de "l'amour en Occident». En effet, les auteur.e.s de chroniques sont des "producteurs culturels [qui] n'ont pas nécessairement un discours idéologique cohérent et construit » (Sapiro, 2007). Leur récit révèle cependant une doxa (Bourdieu, 1997) qui propose depuis leur position doublement dominée (dominé à l'intérieur d'un souschamp littéraire lui-même dominé) un discours relativement révolutionnaire (au sens symbolique). En effet, bien que celui-ci ne défende ni idéologie ni position politique, il révèle une stratégie - ni raison calculatrice, ni déterminations mécaniques de la nécessité économique, ni stratégie consciente, ni détermination automatique " (Dewerpe, 1996) - d'autonomisation par rapport aux principes dominants de définition de l'amour. On peut en effet considérer, en suivant les analyses de Pierre Bourdieu (1980) sur les révolutions symboliques, que la production publique de récits mettant en scène des "amours impossibles » fondés sur la double contrainte qui s'exerce sur les jeunes femmes «issues de minorités non blanches» dans les cités est une forme de réappropriation collective du pouvoir de définition de l'amour romantique selon des principes socialement dominés : ceux des femmes supposées lire ces récits. 


\section{BIBLIOGRAPHIE}

ALBENGA Viviane (2017), S'émanciper par la lecture. Genre, classe et usages sociaux des livres, Rennes, Presses Universitaires de Rennes.

BETTINOTTI Julia (1986), « La Corrida de l'amour : le Roman Harlequin », Montréal, Université du Québec, Les Cahiers du département d'études littéraires, $\mathrm{n}^{\circ} 6$.

BETTINOTTI Julia et Pascale NOIZET (1989) «Le récit en littérature populaire : répétition dans le roman d'amour ", Protée, $\mathrm{n}^{\circ} 2$, p. 35-42.

BIGEY Magali et Sandrine OLIVIER (2010), «Ils aiment le roman sentimental et alors ? Lecteurs d'un « mauvais genre », lecteurs en danger ? » Belphégor, $\mathrm{n}^{\circ}$ 1, [en ligne].

BOURDIEU Pierre (1979), La Distinction, critique sociale du jugement, Paris, Minuit.

BOURDIEU Pierre (1980), «L'identité et la représentation [Éléments pour une réflexion critique sur l'idée de région] », Actes de la recherche en sciences sociales, vol. 35, p. 63-72

BOURDIEU Pierre (1990), «La domination masculine », Actes de la Recherche en Sciences Sociales, $\mathrm{n}^{\circ} 84$, septembre, p. 4-31.

BOURDIEU Pierre (1997), Méditations pascaliennes, Paris, Seuil.

CASTRILLO BUSTAMENTE Concepción (2018), « Entre la abnegación y la autonomía. Disposiciones de género en tensión en la experiencia de los vínculos amorosos heterosexuales », Revista española de Sociología, n² 27, vol. 3, p. 379-394.

CHARPENTIER Isabelle (1995), « Prévenir le péril en la demeure. Les lectures féminines "lascives" : de l'encadrement médical du for intérieur au contrôle social (XVII ${ }^{\mathrm{e}}$-XVIII ${ }^{\mathrm{e}}$ siècles ", dans Claudine HAROCHE (dir.), Le for intérieur, Paris, Presses universitaires de France, p. 305-324.

CHARTIER Roger (1987), Lectures et lecteurs dans la France d'Ancien Régime, Paris, Éditions du Seuil. CHARTIER Roger (1988), « Textes, imprimés, lectures », dans Martine POULAIN (dir.), Pour une sociologie de la lecture, Paris, Éditions du Cercle de la librairie, p. 11-28.

CLAIR Isabelle (2007), « la division genrée de l'expérience amoureuse. Enquêtes dans les cités d'habitat social ", Sociétés et représentations, n² 24, p. 145-160.

CONNELl Raewyn (2014 [1995]), Masculinités. Enjeux sociaux de l'hégémonie, Paris, Éditions Amsterdam.

CONSTANS Ellen (1999), Parlez-moi d'amour : le roman sentimental des romans grecs aux éditions de l'an 2000, Limoges, Presses Universitaires de Limoges.

CoQUilLat Michelle (1988), Romans d'amour, Paris, Éditions Odile Jacob.

COUTRAS Jacqueline (2003), « Territoires du quotidien et espaces asexués. Du voisinage résidentiel aux espaces d'anonymat », Strates, $n^{\circ} 14$, [en ligne le 5 Mars 2013].

CRENSHAW WILLIAMS Kimberlé (2005 [1991]), « Cartographie des marges : Intersectionnalité, politiques de l'identité et violences contre les femmes de couleur », Cahiers du genre, $n^{\circ} 39$, p. 51-82 [ "Mapping the Margins : Intersectionality, Identity Politics, and Violence against Women of Color ", Stanford Law Review, vol. 43, n 6, p. 1241-1299]. 
DAMIAN-GAILLARD Béatrice (2012), « Les romans sentimentaux des collections Harlequin : quelle(s) figure(s) de l'amoureux ? Quel(s) modèle(s) de relation(s) amoureuse(s) ? ", Questions de communication, $\mathrm{n}^{\circ} 20, \mathrm{p} .317-336$.

DEKKER Rudolf (2002), Egodocuments and History, Autobiographical Writing in its Social Context since the Middle Ages, Hilversum, Verloren.

DE SINGLY François (1996), Le Couple, le soi et la famille, Paris, Nathan.

DEWERPE Alain (1996), « La « stratégie » chez Pierre Bourdieu », Enquête [En ligne], 3, URL : http:// journals.openedition.org/enquete/533

DORLIN Elsa (2008) «Introduction. Black feminism Revolution! La Révolution du féminisme noir ! », dans Hazel CARBY et al., Black feminism. Anthologie du féminisme africain-américain, 1975-2000, Paris, L'Harmattan, p. 9-42.

FERHATI Barkahoum (2007), « Les clôtures symboliques des Algériennes : la virginité ou l'honneur social en question », Clio. Femmes, Genre, Histoire [En ligne], 26, p. 169-180.

FLANDRIN Jean-Louis (1982), « La vie sexuelle des gens mariés dans l'ancienne société : de la doctrine de l'Église à la réalité des comportements. ", Communications, n 35, p. 102-115.

GAYMARD Sandrine (2003), La Négociation interculturelle chez les filles franco-maghrébines, Paris, L'Harmattan.

GUILLAUMin Colette (1972), L'Idéologie raciste. Genèse et langage actuel, Paris, Mouton.

HAZEL Carby, «Femme blanche écoute ! Le féminisme noir et les frontières de la sororité », dans Carby HAZEL et al. (2008), Black feminism. Anthologie du féminisme africain-américain, 1975-2000, Paris, L'Harmattan, Bibliothèque du féminisme, p. 87-111.

HELGORSKY Françoise (1985) « Harlequin ou la recherche du grand amour », Communication et langages, $\mathrm{n}^{\circ} 63$, p. 83-98.

HAMEL Christel (2006), « La sexualité entre sexisme et racisme : les descendantes de migrant·e·s du Maghreb et la virginité », Nouvelles Questions Féministes, $\mathrm{n}^{\circ}$ 1, vol. 25, p. 41-58.

HAMMOUCHE Abdelhafid (2013), «Construction sociale et gestion publique de l'altérité », Annuaire de l'EHESS, p. 575-576.

HARCHI Kaoutar (2018), « Appropriation ». Publictionnaire. Dictionnaire encyclopédique et critique des publics. Mis en ligne le 08 novembre 2018. Accès : http://publictionnaire.huma-num.fr/notice/ appropriation/.

HOUEL Annik (1997), Le Roman d'amour et sa lectrice. Une si longue passion. L'exemple Harlequin. Paris/ Montréal, L'Harmattan.

JAMAIN-SAMSON Sandrine et Philippe LIOTARD (2011), « La « Lolita » et la « sex bomb », figure de socialisation des jeunes filles. L'hypersexuation en question. », Sociologie et sociétés, $n^{\circ} 1$, p. 45-71. KAUfMANN Jean-Claude (1993), Sociologie du couple, Paris, Presses Universitaires de France.

KNIBIEHLER Yvonne et al. (1983), De la pucelle à la minette, les jeunes filles de l'âge classique à nos jours, Paris, Temps Actuels.

LACOUE-LABARTHE Isabelle et Sylvie MOUYSSET (2014), « De 'l'ombre légère' à la 'machine à écrire familiale' », CLIO. Histoire, femmes et sociétés [En ligne], n 35, p. 9. Mis en ligne le 01 mai 2014 : http://clio.revues.org/10489. 
LAHIRE Bernard (2004), La Culture des individus. Dissonances culturelles et distinctions de soi, Paris, La Découverte.

LA MOTHE Jacques (1989), Les Mauvais genres, Paris, Éditions du clpcf.

LEMOINE-LUCCIONI Eugénie (1976), Partage des femmes, Paris, Seuil.

MACÉ Éric (2007), « Des ‘minorités visibles’ aux néostéréotypes. Les enjeux des régimes de monstration télévisuelle des différences ethnoraciales ", Journal des anthropologues, Hors-série Identités nationales d'État, p. 69-87.

MARPSAT Maryse (2010), « Écrire la rue : de la survie physique à la résistance au stigmate. - Une analyse textuelle et thématique du journal d'Albert Vanderburg, sans domicile et auteur de blog [archive] », Sociologies, $\mathrm{n}^{\circ}$ 1, p. 195-120.

NADER Laura (2006), « Orientalisme, occidentalisme et contrôle des femmes », Nouvelles Questions

Féministes, $\mathrm{n}^{\circ} 1$, vol. 25, p. 12-24.

NoIRIEL Gérard (1988), « Les jeunes “d'origine immigrée” n'existent pas », dans Bernard LOREYTTE, Les Politiques d'intégration des jeunes issus de l'immigration, Paris, CIEMI-L'Harmattan.

OLIVIER Sandrine (2009), « Les prémices d'une histoire d'amour ou comment écrire un roman sentimental », dans Jacques LA MOTHE (dir.), Mauvais genres, Paris, Éditions du CLPCF, p. 2-25.

PEQUIGNOT Bruno (1991) La Relation amoureuse analyse sociologique du roman sentimental moderne, Paris, L'Harmattan.

PHETERSON Gail (2001[1996]), Le Prisme de la prostitution, Paris, L'Harmattan, [The Prostitution Prism,] Amsterdam, Amsterdam University Press.

REA Andrea et Maryse TRIPIER (2008), « V. Les catégorisations de l'altérité », dans Andréa REA (dir.), Sociologie de l'immigration, Paris, La Découverte, p. 58-71.

RougEMont Denis (1972 [1983]), L'Amour et l'Occident, Paris, Plon.

SAPIRO Gisèle (2007), « Pour une approche sociologique des relations entre littérature et idéologie ", COnTEXTES [En ligne], n². Mis en ligne le 15 février 2007, URL : http:// journals.openedition.org/contextes/165

SHORTER Edwards, (1977), Naissance de la famille moderne, Paris, Seuil.

TISSOT Sylvie (2007), L'État et les quartiers. Genèse d'une catégorie de l'action publique, Paris, Seuil.

\section{NOTES}

1. Cette expression est traduite habituellement par «féminisme noir». Cependant, Elsa Dorlin explique que la désignation du « féminisme africain-américain » dans sa langue d'origine permet de souligner la spécificité de l'histoire politique dans laquelle il a émergé : une société anglosaxonne clivée par le racisme (Dorlin, 2008).

2. « Modèle » renvoie ici à un système social (normes, valeurs) socialement valorisé.

3. Voir à ce sujet Denis Rougemont (1972 [1983]) qui, en France, constitue un exemple paroxysmique de l'idéologie dominante en matière amoureuse et conjugale.

4. Nous reprenons ici la traduction effectuée par Françoise Armangaud des espaces géopolitiques utilisée par Laura Nader (2006) sous les termes «East» et "Weast». Le premier est traduit part «Orient» et le second par «Occident». L'auteure inclut prioritairement dans les pays occidentaux les États-Unis et l'Europe de l'Ouest. 
5. La catégorie « issu.e de l'immigration » pour désigner la population reconnue comme telle en France est problématique (Noiriel, 1988).

6. Les concepts de champ, sous-champ et espace proviennent de la sociologie de la domination (Bourdieu, 1979).

7. Quartiers sensibles, cités, banlieues désignent des quartiers repérés au sein de dispositifs d'action publique à partir de plusieurs indicateurs (insécurité, taux de chômage élevé, échec scolaire, délinquance...) qui conduisent à circonscrire spatialement un ensemble de « problèmes sociaux " qui, en retour, légitiment l'expertise publique (Tissot, 2007).

8. Titre de publication Facebook : «Chronique d'une Cendrillon promise à un prince de Tess ».

9. «Alternance et ambivalence », Enquête [En ligne], 1 | 1985, mis en ligne le 26 juin 2013. URL : http://journals.openedition.org/enquete/39

10. «Aujourd'hui, les romans Harlequin se déclinent entre, d'un côté, des livres de poche : les collections mensuelles, les hors-séries thématiques (Mariage, Noël, Saint-Valentin, sélection des lectrices...), de l'autre, l'édition de romans fictions de grands formats (best-sellers, Mira, Jade, Luna, Red Dress Ink, Spicy), édités tous les deux mois. Il existe également des sagas (L'Héritage des Coltons, Croisière sur l'Alexandra, Les Mac Gregor...) imprimées soit en livres de poche, soit en livres grands formats (dont la plupart deviennent ensuite des livres de poche).» (Damian-Gaillard, 2014, p. 318).

11. Des héros et des hérö̈nes orientaux apparaissent dans les romans Harlequin mais ils se distinguent fondamentalement de ceux et celles des Thug Love par le fait que ces derniers proviennent de l'immigration et vivent dans les « cités ».

12. Les termes «féminité » et «masculinité » renvoient dans ce texte à la construction sociale des identités sexuées.

13. Soulignons que François de Singly (1996) a élaboré une théorie de l'identité individuelle dans le couple, à partir d'un matériau exclusivement constitué de romans et de films. Une telle démarche ne peut être considérée comme identique à l'enquête de terrain à proprement parler mais elle permet de dégager des discours sociaux typifiés.

14. Nous remercions Mathieu Ferry qui a effectué l'extraction et la classification thématique des données.

15. Bien que nos analyses nous conduisent à faire l'hypothèse qu'il s'agit de femme, nous ne pouvons l'affirmer en l'absence d'échanges directs avec leurs auteur.e.s.

16. Cet aspect de l'analyse n'est pas traité dans cet article. Nous avons toutefois tenu compte (sans en faire une analyse systématique) des commentaires faits par les lectrices (les commentaires sont écrits au féminin).

17. La collection intègre dans son schéma une nouvelle réalité sociale, soit la nouvelle importance du monde arabe dans le monde occidental depuis la crise du pétrole (Bettinoti, Noizet, 1989).

\section{RÉSUMÉS}

Cet article propose une analyse de chroniques amoureuses publiées sur internet, appelées « Thug Love ». Elles mettent en scène des histoires d'amour de jeunes filles et de jeunes garçons de familles originaires d'Afrique du Nord ou de Turquie et vivant dans des « cités ». Ce travail a pour objet de montrer en quoi les « Thug » entrent à la fois en résonnance avec la structure narrative 
des romans Harlequin et prennent appui (en les renforçant) sur des représentations ordinaires des relations entre filles et garçons de familles musulmanes en France. D'une manière plus générale il s'agit de montrer comment l'idéologie raciste imprègne le modèle patriarcal de l'amour hétérosexuel dans les œuvres étudiées.

This paper intends to analyse web published love chronicles, called "Thug Love". They romanticize love stories between North African and Turkey born suburban young girls and boys. This work aims at showing how the "Thug" both resonate with the narrative structure of the Harlequin novels and lean on (while strengthening them) typical representations of the relationship between boys and girls descending from Muslim families in France. Broadly speaking, it deals with demonstrating how the racist ideology flows through the androcentric model of the heterosexual love in the studied works.

\section{INDEX}

Mots-clés : thug love, romance en série, hétérosexualité, sexe, classe, racialisation, littérature populaire

Keywords : thug love, heterosexuality, sex, class, racialization, popular literature

\section{AUTEURS}

\section{MÉLIE FRAYSSE}

Mélie Fraysse est chercheure associée au CreSco (université Paul Sabatier Toulouse 3). Ses travaux portent sur la fabrique du genre dans les médias mais aussi sur les socialisations professionnelles des journalistes et notamment sur leurs effets dans la pratique journalistique. Elle poursuit actuellement ses travaux sur les rapports sociaux de sexe en s'intéressant à la gouvernance des femmes dans les instances sportives.

\section{MARIE-CARMEN GARCIA}

Marie-Carmen Garcia est sociologue, Professeure des universités à l'Université Paul SabatierToulouse 3. Chercheuse au laboratoire $\mathrm{CreSco}$, ses travaux portent sur les socialisations de genre dans différents espaces de la vie sociale : politique, éducation, sport, loisirs, travail. Elle s'intéresse particulièrement à la production et l'incorporation des normes émotionnelles et sexuelles selon la classe et le sexe. En 2016, elle a publié Amours clandestines (PUL) où elle analyse les représentations et pratiques des femmes et des hommes impliqués dans une relation amoureuse cachée. Elle poursuit actuellement ses travaux dans ce domaine tout en élargissant ses questionnements en sociologie des émotions aux socialisations professionnelles. 